\title{
The Effects of Boundary Turbulence on Canyon Flows Forced by Periodic Along-Shelf Currents
}

\author{
DON L. BOYER \\ Environmental Fluid Dynamics Program, Department of Mechanical and Aerospace Engineering, Arizona State University, \\ Tempe, Arizona \\ JoEl SOMMERIA \\ Equipe Coriolis, Laboratoire des Ecoulements Geophysiques et Industriels, Grenoble, France \\ Andjelka Srdic Mitrovic and V. K. Chaitanya Pakala \\ Environmental Fluid Dynamics Program, Department of Mechanical and Aerospace Engineering, Arizona State University, \\ Tempe, Arizona \\ Sergey A. SMirnov \\ Mechanical Engineering Department, Texas Tech University, Lubbock, Texas \\ DiETER ETLING \\ Institut Für Meteorologie und Klimatologie, University of Hannover, Hannover, Germany
}

(Manuscript received 11 February 2004, in final form 7 September 2005)

\begin{abstract}
Previous laboratory experiments and associated numerical models of laminar flows forced by oscillatory, along-shelf background currents are extended to include some of the effects of boundary-generated turbulence. The experiments are conducted in the 13-m-diameter rotating-flow facility in Grenoble, France. Two pairs of case studies, one at a large forcing velocity (designated as FAST) for which the boundary layers are fully turbulent during part of the flow cycle and one at relatively smaller forcing (SLOW) for which transitional boundary layers are operative at the higher speeds of the background flow, are conducted. Smooth and artificially roughened boundaries are considered, respectively, for each of these pairs. Phaseaveraged and time-mean velocity, vertical vorticity, and horizontal divergence fields are found to be qualitatively similar to those of previous laminar experiments. The similarities in the time-mean fields are that (i) within the canyon they are dominated by cyclonic vorticity with maxima centered near the shelf break; (ii) within and in the vicinity of the canyon the general circulation pattern includes a net transport into the canyon through its mouth, a net upwelling in the canyon interior, a transport away from the canyon over the shelf break along both sides of the canyon, and, by inference, a return flow to the deep ocean; and (iii) the interior time-mean flow is characterized by a well-defined coastal current whose axis follows the shelf in the vicinity of the shelf break, with the coast on the right. It is found that the measurements of the characteristic speed of the residual or time-mean flow within the canyon for the transitional and fully turbulent experiments do not follow the scaling law derived earlier for laminar experiments. An alternative scaling analysis for large-Reynolds-number flows is thus derived. Although sufficient numbers of experiments are not available to test the hypothesis fully, the measurements available for the fully turbulent flows are consistent with the theory advanced.
\end{abstract}

Corresponding author address: Dr. Don L. Boyer, Environmental Fluid Dynamics Program, Department of Mechanical and Aerospace Engineering, Arizona State University, P.O. Box 876106, Tempe, AZ 85287.

E-mail: don.boyer@asu.edu

(C) 2006 American Meteorological Society 


\section{Introduction}

The long-range goal of this research is to establish a physical modeling approach to coastal and other oceanic systems that can effectively serve the numerical modeling community in the development and benchmarking of ocean models. In this paper we report on time-dependent and time-mean currents developed in and near isolated canyons for cases in which the bottom boundary layer is either in transition from laminar to turbulent flow or is fully turbulent for a portion of the period of the oscillation. The fluid is rotating and stratified and is forced by an oscillatory along-slope background current. The principal objective of the study is to investigate in what ways, and to what degree, boundary turbulence and/or transition to turbulence influences the resulting flow fields.

Five experiments will be discussed; four of these are new and one, for comparison purposes, has been discussed previously by Pérenne et al. (2001, hereinafter PHB) and as experiment 01 in Boyer et al. (2004, hereinafter BHP). In the current paper, this experiment will be referred to as ASU-01. Owing to the extensive discussion in those articles concerning the motivation and background for the present study, only a few brief introductory remarks are given here.

Few field studies reporting on aspects of the general circulation patterns within, and in the vicinity of, submarine canyons appear in the literature. A good starting point for the interested reader is the study by Hickey (1997) in which she reports on field observations of upwelling events in and near Astoria Canyon. This article is used as a guide to define the values and ranges of some of the oceanic parameter values of in- terest (see our Tables 1 and 2) and to compare the laboratory results, at least qualitatively, with ocean observations.

A description of the physical system, the governing parameters, the experimental facility and techniques employed, and outlines of the analyses to be used are given in section 2 . The laboratory observations and measurements and the interpretation of the data are given in section 3. A summary, discussion, and concluding remarks are presented in section 4 .

\section{Physical system, parameters, scaling, and data analysis}

\section{a. Physical system}

A schematic diagram of the physical system is given in Fig. 1. The facility is a circular tank containing an axisymmetric continental shelf, slope, and deep-ocean model incised by a single isolated canyon. The depth contours of the topography are given by Boyer et al. (2000, hereinafter BZP). The scaling factor for all dimensions between the small and large facilities is 7.2. The canyon is characterized by its length $2 L$ measured along the canyon axis from the point where the canyon intersects the horizontal shelf to the radius at which the sloping region reaches the deep-water floor. The characteristic width $W$ is defined as the distance along the shelf break between the points where the canyon intersects the shelf. Other geometrical parameters are defined in Table 1 and indicated in Fig. 1.

The canyon, shelf, and slope are either smooth or covered with cubical roughness elements of side $d$ placed along lines of constant radius on the shelf and in

TABLE 1. Dimensional parameters; quantities with asterisks are not independent of the other parameters. The ASU-01 experiment was reported as expt 01 in BHP.

\begin{tabular}{|c|c|c|c|c|}
\hline Parameter & Astoria Canyon & ASU-01 & Grenoble SLOW & Grenoble FAST \\
\hline$R_{C}$ (radius of the coast) & - & $35 \mathrm{~cm}$ & $2.53 \mathrm{~m}$ & $2.53 \mathrm{~m}$ \\
\hline$R_{S}$ (radius at the shelf break) & - & $55 \mathrm{~cm}$ & $3.97 \mathrm{~m}$ & $3.97 \mathrm{~m}$ \\
\hline$R_{T}$ (radius of the test tank $)$ & - & $90 \mathrm{~cm}$ & $6.50 \mathrm{~m}$ & $6.50 \mathrm{~m}$ \\
\hline$h_{S}($ depth over the shelf $)$ & $150 \mathrm{~m}$ & $2.5 \mathrm{~cm}$ & $0.18 \mathrm{~m}$ & $0.18 \mathrm{~m}$ \\
\hline$h_{D}(\max$ depth $)$ & $600 \mathrm{~m}$ & $12.5 \mathrm{~cm}$ & $0.90 \mathrm{~m}$ & $0.90 \mathrm{~m}$ \\
\hline$W$ (width of the canyon) & $7 \mathrm{~km}$ & $20 \mathrm{~cm}$ & $1.44 \mathrm{~m}$ & $1.44 \mathrm{~m}$ \\
\hline$L$ (length of the canyon) & $15 \mathrm{~km}$ & $15 \mathrm{~cm}$ & $0.94 \mathrm{~m}$ & $0.94 \mathrm{~m}$ \\
\hline$D$ (size of the roughness) & - & - & $0.03 \mathrm{~m}$ & $0.03 \mathrm{~m}$ \\
\hline$S$ (spacing of roughness) & - & - & $0.00,0.15 \mathrm{~m}$ & $0.00,0.15 \mathrm{~m}$ \\
\hline$f$ (Coriolis parameter) & $0.0001 \mathrm{~s}^{-1}$ & $0.50 \mathrm{~s}^{-1}$ & $0.093 \mathrm{~s}^{-1}$ & $0.25 \mathrm{~s}^{-1}$ \\
\hline$N$ (Brunt-Väisälä frequency) & $0.005 \mathrm{~s}^{-1}$ & $2.5 \mathrm{~s}^{-1}$ & $0.46 \mathrm{~s}^{-1}$ & $0.46 \mathrm{~s}^{-1}$ \\
\hline$\omega$ (forcing frequency) & $0.000015 \mathrm{~s}^{-1}$ & $0.26 \mathrm{~s}^{-1}$ & $0.048 \mathrm{~s}^{-1}$ & $0.125 \mathrm{~s}^{-1}$ \\
\hline$\nu$ (molecular viscosity) & $0.01 \mathrm{~cm}^{2} \mathrm{~s}^{-1}$ & $0.01 \mathrm{~cm}^{2} \mathrm{~s}^{-1}$ & $0.01 \mathrm{~cm}^{2} \mathrm{~s}^{-1}$ & $0.01 \mathrm{~cm}^{2} \mathrm{~s}^{-1}$ \\
\hline$u_{0}$ (amplitude of forcing) & $10 \mathrm{~cm}^{-1}$ & $1 \mathrm{~cm} \mathrm{~s}^{-1}$ & $1.33 \mathrm{~cm} \mathrm{~s}^{-1}$ & $5.0 \mathrm{~cm} \mathrm{~s}^{-1}$ \\
\hline$X=2 u_{0} / \omega(\text { excursion })^{*}$ & $13 \mathrm{~km}$ & $7.6 \mathrm{~cm}$ & $0.54 \mathrm{~m}$ & $0.8 \mathrm{~m}$ \\
\hline$L_{D}=N h_{D} / f(\text { Rossby radius })^{*}$ & $30 \mathrm{~km}$ & $62.0 \mathrm{~cm}$ & $4.5 \mathrm{~m}$ & $1.7 \mathrm{~m}$ \\
\hline
\end{tabular}


TABLE 2. Dimensionless parameters; quantities with asterisks are not independent of the other parameters.

\begin{tabular}{lcccc}
\hline \hline \multicolumn{1}{c}{ Parameter } & Astoria Canyon & ASU-01 & Grenoble SLOW & Grenoble FAST \\
\hline$R_{C} / R_{T}$ & - & 0.39 & 0.39 & 0.39 \\
$R_{S} / R_{T}$ & - & 0.61 & 0.61 & 0.61 \\
$h_{D} / W$ & 0.09 & 0.625 & 0.625 & 0.625 \\
$W / L$ & 0.47 & 1.33 & 1.33 & 1.33 \\
$W / R_{T}$ & - & 0.22 & 0.22 & 0.22 \\
$h_{S} / h_{D}$ & 0.25 & 0.20 & 0.20 & 0.20 \\
$d / h_{D}$ & - & 0.00 & $0.0,0.033$ & 0.033 \\
$S / h_{D}$ & - & 0.00 & 0.0 .17 & 0.17 \\
$\operatorname{Re}_{d}=u_{0} d / \nu$ & - & 0.00 & $0.00,400$ & 1500 \\
$\operatorname{Re}_{\delta}=u_{0}(2 / \nu f)^{1 / 2}$ & 14,100 & 20 & 61.7 & 0.14 \\
$\operatorname{Ro}^{\prime}=u_{0} /(f W)$ & 0.14 & 0.10 & 0.10 & 0.14 \\
$\mathrm{Bu}^{2}\left(N^{2} h_{D}^{2}\right) /\left(f^{2} W^{2}\right)$ & 18 & 9.8 & $3.0 \times 10^{-4}$ & 1.36 \\
$\mathrm{Ek}=\nu /\left(f h_{S}^{2}\right)$ & $4.4 \times 10^{-7}$ & $3.2 \times 10^{-3}$ & 0.52 & $1.0 \times 10^{-4}$ \\
$\operatorname{Ro}_{t}=\omega / f$ & 0.15 & 0.52 & 0.38 & 0.50 \\
$X / W^{*}$ & $0.4,0.56$ & 0.38 & & 0.55 \\
\hline
\end{tabular}

rows along the depth contours on the slope and canyon. The rows and along-shelf separation distances $S$ are set at $5 d$. These models are designated as smooth (S) and rough $(\mathrm{R})$, respectively. They are placed in the $13-\mathrm{m}-$ diameter tank mounted on a turntable operated by the Laboratoire des Ecoulements Geophysiques et Industriels (LEGI) in Grenoble, France.

The tank is filled while rotating with a linearly stratified fluid using standard laboratory techniques (saltwater); initially, the fluid thus has a constant BruntVäisälä (buoyancy) frequency $N$ and an assumed constant kinematic viscosity $\nu$. The rotation rate is specified by the Coriolis parameter $f=2 \Omega$, where $\Omega$ is the rate of rotation. Figure 2 is a photograph of the Grenoble facility in which the white dots on the dark background are the roughness elements and the canyon can be seen in the foreground. At the time when the fluid reaches a suitable state of solid body rotation, the forcing flow is initiated by modulating the tank rotation rate about its background value by the function $\Delta \Omega$ $\sin (\omega t)$, where $\Delta \Omega$ and $\omega$ are the amplitude and frequency of the modulation, respectively. An observer rotating with the tank thus sees an oscillating, alongisobath current relative to the canyon. The characteristic velocity of the background flow $u_{0}$ is taken as the amplitude of the oscillating fluid as measured at the shelfbreak radius $R_{S}$; that is, $u_{0}=R_{S} \Delta \Omega$. Owing to the oscillatory nature of the turntable rotation rate, some "nonoceanic" terms appear in the relations for Newton's second law. These terms are readily included in any theoretical or numerical model of the system, and thus the laboratory results can be used as a benchmark for ocean models (see BZP).

Particle image velocimetry (PIV) is used to obtain time series data of horizontal velocity fields in a $210 \mathrm{~cm}$ $\times 160 \mathrm{~cm}$ rectangular area at selected vertical levels.
Given the resolution of the camera $(768 \times 484$ pixels $)$ and the typical size of the interrogation cell $(24 \times 20$ pixels), the spatial resolution of the measurement is approximately $6.5 \mathrm{~cm} \times 6.5 \mathrm{~cm}$.

The flow fields for the present physical system have been shown to spin up in one to two oscillation cycles for laminar flows (see BZP). The same spinup time is used for the present experiments; data are taken beginning at the 11th rotation period extending through the 20th. By analyzing the data for small increments of time, the "instantaneous" horizontal motion field can be obtained (e.g., see Fig. 6, below). Time series data for fixed Eulerian positions can then be readily obtained from these data (e.g., see Fig. 5, below). To find the phase-averaged behavior, an ensemble average of the data from the 10 oscillations is carried out (e.g., see Fig. 4, below). Last, these ensemble-averaged findings are time averaged to determine the time-mean flow (e.g., see Fig. 7, below).

\section{b. Parameters}

The physical system described can be characterized by a Rossby number Ro $=u_{0} / f W$, a temporal Rossby number $\operatorname{Ro}_{t}=\omega / f$, a Burger number $\mathrm{Bu}=\left(N^{2} h_{D}^{2}\right) /$ $\left(f^{2} W^{2}\right)$, and an Ekman number Ek $=\nu / f h_{S}^{2}$. When artificial roughness elements are used, the Reynolds number $\mathrm{Re}_{d}$, based on the size of the roughness elements, is also important and is defined as $\operatorname{Re}_{d}=u_{0} d / \nu$. There are many geometric parameters, all of which are held fixed in the experiments. These include parameters involving the large-scale features of the canyon and its local environment (horizontal aspect ratio $L / W$, vertical aspect ratio $h_{D} / W$, fractional depth $h_{S} / h_{D}$, normalized roughness $d / h_{D}$, and normalized spacing $S / h_{D}$ ) and other basin-scale parameters, including $R_{C} / R_{T}, R_{S} / R_{T}$, and $W / R_{T}$. 

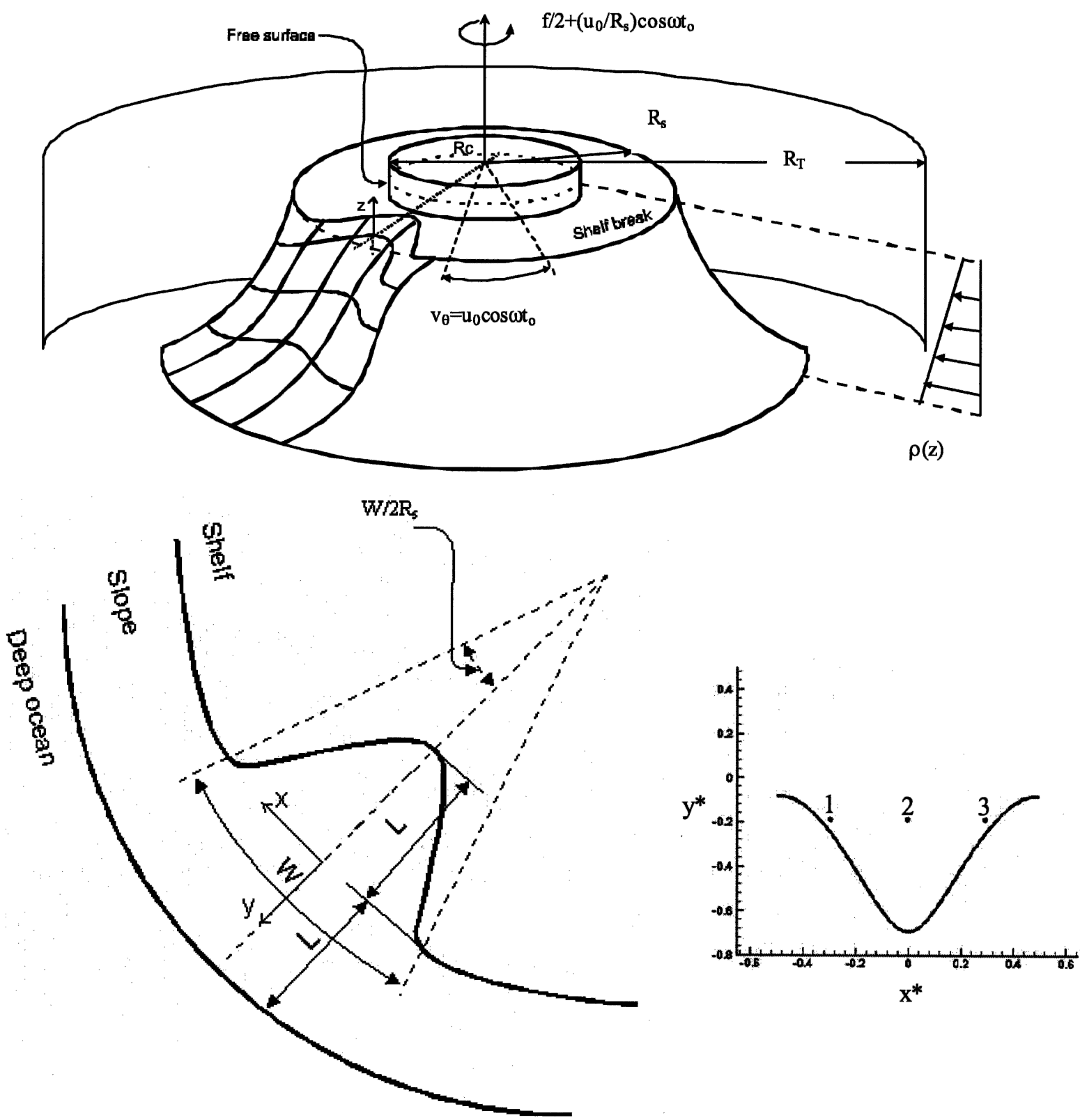

FIG. 1. Physical system. The lower-right-side insert indicates the locations of the Eulerian measurements depicted in Figs. 5a-c.

Two parameters, important for the interpretation of the data, are the Rossby radius of deformation $R_{d}=$ $N h_{D} / f$ (the Burger number is obtained by normalizing this parameter by the width of the canyon) and a normalized tidal excursion $X^{*}=X / W$. The latter is introduced as the dimensionless distance that an undisturbed fluid parcel moves in one oscillation cycle; that is, the normalized tidal excursion $X^{*}=2 \mathrm{Ro} / \mathrm{Ro}_{t}$, where $X^{*}$ is not an independent parameter. The system parameters are defined and values given in Table 1 (dimensional) and Table 2 (dimensionless); see also Fig. 1.

Recognizing that the criteria for the transition to tur- bulence for this complex physical system are unknown, we employ, as a guide, Lilly's (1966) criterion for the instability of a homogeneous incompressible fluid flowing over a flat surface in a rotating frame; that is,

$$
\operatorname{Re}_{\delta}=\left(2 u_{0}^{2} / \nu f\right)^{1 / 2} \geq 55
$$

This criterion is considered the upper limit on $\mathrm{Re}_{\delta}$ because it is anticipated that the perturbations added by complex topography will tend to make the boundary layers more unstable at smaller Reynolds numbers. Caldwell and Van Atta (1970) conducted laboratory 


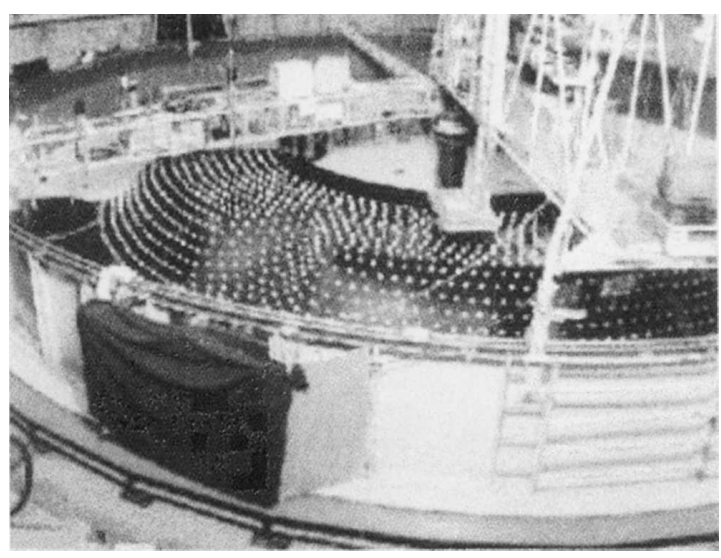

FIG. 2. Coriolis turntable, Grenoble, France: the white "dots" on the black background are the roughness elements. The canyon is just below and to the left of the center of the photograph.

experiments that support the Lilly theory in that instabilities were noted at $\operatorname{Re}_{\delta}=56.7$, but these were in the form of rolls with longitudinal axes along the streamwise direction. Fully developed turbulence was observed only for $\mathrm{Re}_{\delta}>148$. In separate experiments we have measured directly the bottom friction law by spinup and spindown for a homogeneous fluid layer over a flat bottom. The square of the friction velocity along the tank floor $u_{*}^{2}$ was, indeed, found to change at $\mathrm{Re}_{\delta} \approx 150$ from the laminar Ekman linear friction law to a turbulent law, with $u_{*}^{2} \approx 0.005 U^{2}$ (Ferrero et al. 2005).

\section{c. Scaling of time-mean flow and data analysis}

The discussions to follow are facilitated by defining a control volume for the canyon, that is, an imaginary surface encompassing the canyon through which water is free to move (see Fig. 3). The sides of the control volume above the shelf break are defined by the intersection of the shelf with the canyon and a straight line across the canyon mouth (see Fig. 3c). At each level below the shelf break the canyon walls serve as the inner boundary with the outer boundary being the straight line between the extremities of the canyon mouth at that level (see Fig. 3b).

To better understand the underlying physics of the system under consideration, a scaling analysis of the time-mean or rectified flow is discussed. Such an analysis was given in BHP for laminar flow, that is, for low Reynolds numbers. The analysis here employs the same hypothesis used by BHP; that is, the time-mean flow $\bar{u}$ results from a balance between vorticity generation by vortex stretching and dissipation by wall friction. As vortex tubes advect across the shelf break, the fluid parcels stretch but the stretching is limited by the back-
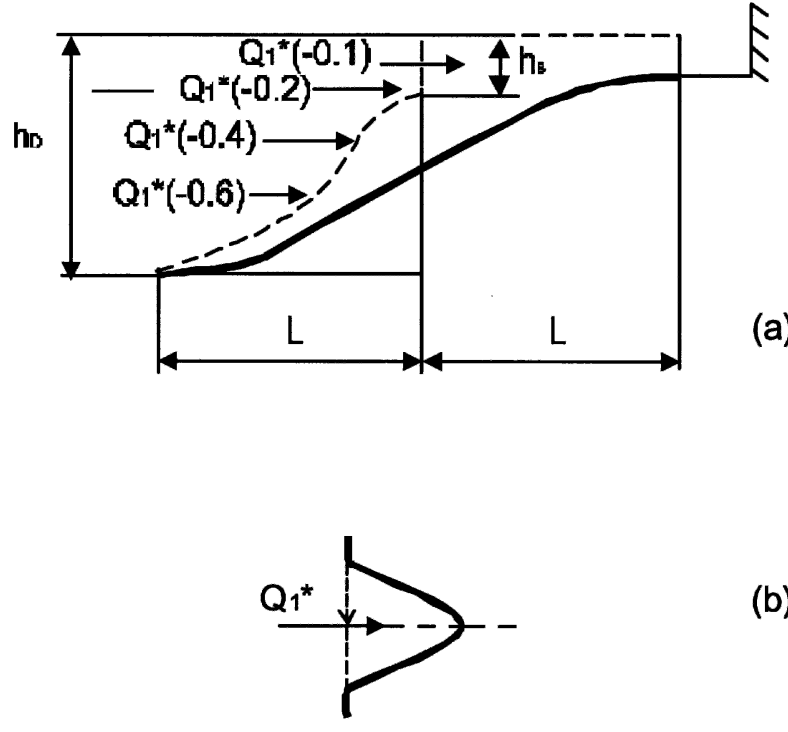

(b)

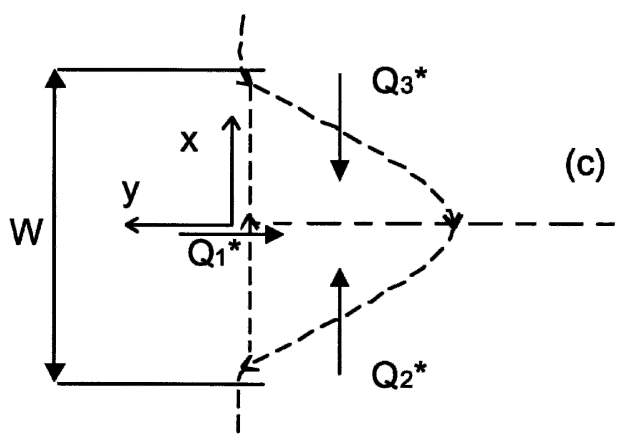

FIG. 3. Schematic diagram of control volume: (a) vertical cross section along the axis of the canyon, (b) plan view at $z^{*}=-0.4$ level, and (c) plan view at $z^{*}=-0.2$, or shelfbreak, level.

ground stratification. The maximum elongation is estimated as the distance a fluid parcel would move vertically downward should all of its kinetic energy be transformed into potential energy in the stratified medium (i.e., $\left.\Delta z \sim u_{0} / N\right)$. Then, using the principle of conservation of potential vorticity for the vortex tubes, a scaling for the relative cyclonic vorticity induced by advection into the control volume is obtained. The vorticity $Z$ produced during one oscillation cycle, horizontally integrated over the canyon, scales as (see BHP)

$$
Z \propto \frac{u_{2}^{0} L f}{h_{S} \omega N} .
$$

This vortex stretching is not affected by the presence of turbulence so that vorticity production is the same for both the laminar and the turbulent flows.

Expressing the bottom shear stress as $\tau=\rho u_{*}^{2}$, the 
total loss of integrated vorticity by bottom friction during one oscillation period is

$$
D=\frac{u_{*}^{2} L}{h_{S} \omega} .
$$

Equating the dissipation $D$ with the production $Z$ yields

$$
\frac{u_{0}^{2} f}{N}=u_{*}^{2}
$$

With the classical laminar Ekman friction law $u_{*}^{2}=$ $(\nu f / 2)^{1 / 2} \bar{u}$, the BHP prediction for the time-mean flow $\bar{u}$ is obtained:

$$
\frac{\bar{u}}{u_{0}}=K \frac{f}{\omega} \frac{u_{0} f^{1 / 2}}{N \nu^{1 / 2}} \equiv K \frac{\mathrm{Ro}}{\left(h_{S} / h_{D}\right) \mathrm{Ro}_{t} \mathrm{Bu}^{1 / 2} \mathrm{Ek}^{1 / 2}} \equiv K \lambda .
$$

The ratio $\bar{u} / u_{0}$ of the time-mean flow to the forcing velocity is proportional to the parameter $\lambda$ defined in (2.5) in terms of either dimensionless or dimensional parameters. The coefficient of proportionality $K$ should depend on the canyon geometry, on the ratio $\mathrm{Ro}_{t}$ of the forcing frequency to the Coriolis frequency, and on the precise definition of the scale $\bar{u}$. The laboratory experiments and numerical model results conducted for laminar flows are in good agreement with (2.5) (see Fig. 9, below).

For turbulent flows, it is well known that the dissipation will depend on the nature of the turbulence, with the kinematic viscosity not being of zeroth-order importance. We may then reasonably assume that with an eddy viscosity $\nu_{t}$ scaling like the dominant velocity $u_{0}$ we write the bottom stress as $\nu_{t} \bar{u} / \delta$, where $\delta$ is a boundary layer thickness and $\nu_{t} \sim u_{0} l$. This yields

$$
u_{*}^{2}=C_{t} u_{0} \bar{u},
$$

where $\mathrm{C}_{t}$ is a nondimensional constant. Then (2.5) is replaced by the scaling

$$
\frac{\bar{u}}{u_{0}}=K_{t} \frac{f}{N},
$$

where $K_{t}$ is a nondimensional factor, possibly depending on the frequency ratio $\omega / f$, the surface roughness, and geometrical aspect ratios.

A mean vertical upwelling velocity can be estimated from the Ekman pumping related to the bottom friction. This vertical velocity is associated by mass conservation to a transverse nongeostrophic horizontal velocity $u_{\perp}$, with $\bar{w} W \approx u_{\perp} h_{S}$. The friction in the bottom layer is transmitted to the bulk fluid by the effect of the Coriolis force on this transverse nongeostrophic veloc- ity $u_{\perp}$, so that $f u_{\perp} h_{s}=u_{*}^{2}$. Combining these two relations yields $\bar{w} \approx u_{*}^{2} /(f W)$ or, using (2.4),

$$
\frac{\bar{w}}{u_{0}}=\gamma \frac{u_{0}}{N W} .
$$

where $\gamma$ is a dimensionless coefficient.

In the experiments we define the vertical distribution of the observed normalized characteristic horizontal speed (designated as horizontal speed below) of the time-mean flow $(u, v)$ in the canyon control volume (Fig. 3) as $\bar{u}_{\text {obs }}\left(z^{*}\right) / u_{o}$; that is,

$$
\frac{\bar{u}_{\mathrm{obs}}\left(z^{*}\right)}{u_{0}}=\left[\frac{1}{A^{*}\left(z^{*}\right)} \oiint \frac{\left(u^{2}+v^{2}\right)}{u_{0}^{2}} d x^{*} d y^{*}\right]^{1 / 2},
$$

where $A^{*}\left(z^{*}\right)$ is the normalized area at the depth $z^{*}$. To test the scaling of relation (2.5) or (2.7), we take the horizontal speed as obtained from (2.9) at the shelf break, that is, $\bar{u}_{\mathrm{obs}}\left(z^{*}=-0.2\right) / u_{0}$.

While the time-mean vertical velocity averaged over a horizontal cross section of the control volume cannot be measured directly, it can be estimated as follows. We first define the normalized volume transports of the time-mean flow per unit depth into the control volume through the canyon mouth as $Q_{1}^{*}\left(z^{*}\right)$, across the left side of the canyon facing toward the deep water as $Q_{2}^{*}\left(z^{*}\right)$, and across the right side (see Fig. 3) as $Q_{3}^{*}\left(z^{*}\right)$; that is,

$$
\begin{aligned}
& Q_{1}^{*}\left(z^{*}\right)=-\frac{1}{u_{0} W} \int_{-W / 2}^{W / 2} v(x, y) d x \\
& Q_{2}^{*}\left(z^{*}\right)=\frac{1}{u_{0} W}\left[\int_{-W / 2}^{0} v(x, y) d x+\int_{y_{h}}^{y_{m}} u(x, y) d y\right]
\end{aligned}
$$

and

$Q_{3}^{*}\left(z^{*}\right)=\frac{1}{u_{0} W}\left[\int_{0}^{W / 2} v(x, y) d x-\int_{y_{h}}^{y_{m}} u(x, y) d y\right]$,

respectively, where $y_{m}$ and $y_{h}$ are the $y$ coordinates of the mouth and the head of the canyon, respectively; because $\left(Q_{2}^{*}, Q_{3}^{*}\right)$ can only be evaluated above the shelf break, these transports are taken as zero elsewhere. Because there can be no flow into the canyon from below the model floor, the total normalized upwelling (volume per unit time) through a horizontal cross section of the control volume is given by

$$
Q_{Z^{*}}^{*}\left(z^{*}\right)=\int_{-1.0}^{z^{*}}\left[Q_{1}^{*}\left(z^{* \prime}\right)+Q_{2}^{*}\left(z^{* \prime}\right)+Q_{3}^{*}\left(z^{* \prime}\right)\right] d z^{* \prime} .
$$


The normalized, space-averaged, time-mean vertical speed $\bar{w}^{*}\left(z^{*}\right)=\left(W / h_{D}\right)\left[w(z) / u_{0}\right]$ can then be determined as

$$
\frac{\bar{w}\left(z^{*}\right)}{u_{0}}=\frac{Q_{z^{*}}^{*}\left(z^{*}\right)}{A^{*}\left(z^{*}\right)}
$$

\section{Observations, measurements, and interpretation}

Four new experiments were conducted. These included two forcing velocity amplitudes $u_{0}$ (1.33, 5.0) $\mathrm{cm} \mathrm{s}^{-1}$, defined as (SLOW, FAST) and, for each, two surface characteristics, designated as smooth (S) and rough $(\mathrm{R})$ (see Tables 1 and 2 ).

\section{a. Time-dependent flows}

The periodic forcing leads, as should be expected, to periodic responses. Figures $4 \mathrm{a}-\mathrm{c}$ and $4 \mathrm{~d}-\mathrm{f}$ depict the phase-averaged velocity and horizontal divergence fields for the FAST-R experiment for phases A (maximum velocity from left to right) and $C$ (maximum velocity from right to left), respectively. Figures $4 \mathrm{a}$,d; Figs. 4b,e; and Figs. 4c, f illustrate the elevations at middepth on the shelf $\left(z^{*}=-0.1\right)$, on the shelf break $\left(z^{*}\right.$ $=-0.2)$, and in the canyon $\left(z^{*}=-0.4\right)$, respectively. The most striking feature of Fig. 4 is the asymmetries in the velocity fields that are of the same speed but are $180^{\circ}$ out of phase; for example, compare and contrast Figs. 4a,d; Figs. 4b,e; and Figs. 4c,f.

The asymmetric nature of the flow can also be noted by examining Eulerian time series data obtained at selected locations in the flow field. Figure 5 (top to bottom) shows measurements of the velocity components $\left(u^{*}, v^{*}\right)$ at the shelfbreak level for the positions indicated as 1, 2, and 3, respectively, in Fig. 1. The velocity components are seen to be roughly $180^{\circ}$ out of phase in Fig. 5 (top) and in phase in Fig. 5 (bottom); the latter is remarkable in the sense that the $(u, v)$ components are almost identical, implying that the fluid at this location is oscillating along the shelf break at almost precisely a $45^{\circ}$ angle. It is apparent from the top and middle panels of Fig. 5 that the time mean of the $\left(u^{*}, v^{*}\right)$ velocity component will be into the canyon on the left side of the canyon mouth and out on the right. This in turn implies that there will be a time-mean flow into the canyon along the left side of the canyon and a timemean flow out of the canyon on the right, respectively.

The flow asymmetries can be understood, in part, by a consideration of the vertical component of vorticity experienced by fluid parcels advecting into the canyon at the level of the shelf break. Consider, first, fluid parcels passing over the shelf break at some central region between the canyon head and mouth. In moving from left to right across the left side of the canyon they will be stretched vertically until buoyancy captures the stretching as described above. This leads to the long thin region of positive divergence in Fig. 4b. One also notes a thin region of negative divergence deeper into the canyon that parallels the line of positive divergence. This region owes to the advection of anticyclonic vorticity entering the canyon through the left side of the mouth. This interaction of fluid parcels having vorticity of opposite signs "neutralizes" the relative vorticity of fluid parcels entering the canyon from the left. As such, when these parcels exit on the right side of the canyon, they follow the depth contours as apparent in Figs. 4b,c.

Similarly, along the right side of the canyon, fluid parcels passing over the slope at the shelf break again attain cyclonic vorticity by vortex stretching. Here, however, the fluid entering the canyon along the right side of the mouth also has cyclonic vorticity. These fluid parcels being strongly cyclonic are able to overcome the tendency to veer to the right (anticyclonically) and, indeed, move upward upon encountering the canyon wall (see Figs. 4e,f). Thus, no second belt of anticyclonic vorticity is observed along the right side of the canyon because there is no local source of anticyclonic vorticity.

The Burger number in the FAST-R experiment is 1.36. This is considered a weakly stratified experiment, and thus some evidence of the depth independence of the motion field (i.e., Taylor-Proudman columns) is expected. This effect is exhibited in Figs. 4b,c where one notes that the time-mean streamlines tend to follow the canyon depth contours at all observation levels, while Figs. 4d-f show the tendency for the mean flow to advect from the upper right to the lower left across the canyon interior.

\section{b. Evidence for the presence of turbulence}

The present experiments were aimed at extending earlier work done on laminar flows to ones associated with boundary turbulence. The experiments were designed to have one set (FAST-S, -R) to be turbulent, based on the Lilly (1966) and Caldwell and Van Atta (1970) studies, and the other set (SLOW-S, -R) to be transitional, based on this same work. The PIV method used for obtaining the velocity fields has a spatial resolution of about $6.5 \mathrm{~cm}$. This resolution, while adequate to monitor interior motions as described above, is not sufficient to interrogate the bottom boundary layers where most of the turbulent dissipation is expected. The present data thus do not allow relating the structure of the bottom boundary layers to the resulting interior flows; an understanding of this boundary layerinterior flow interaction problem is vitally important in 
(a)

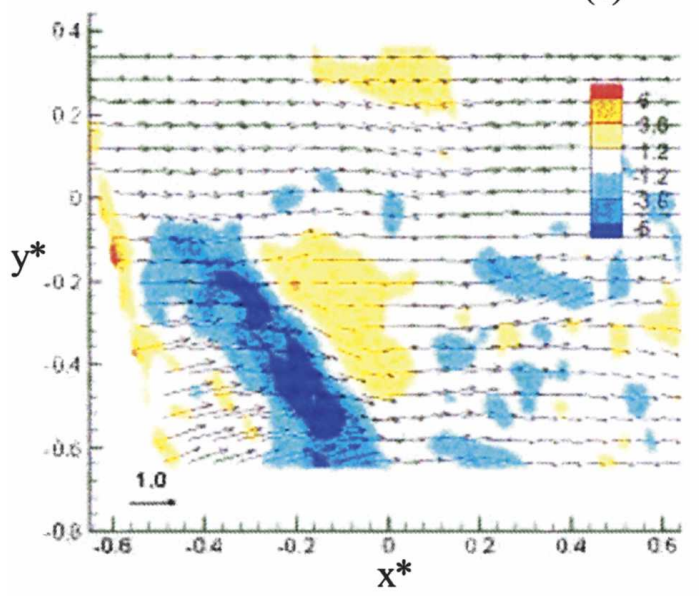

(b)
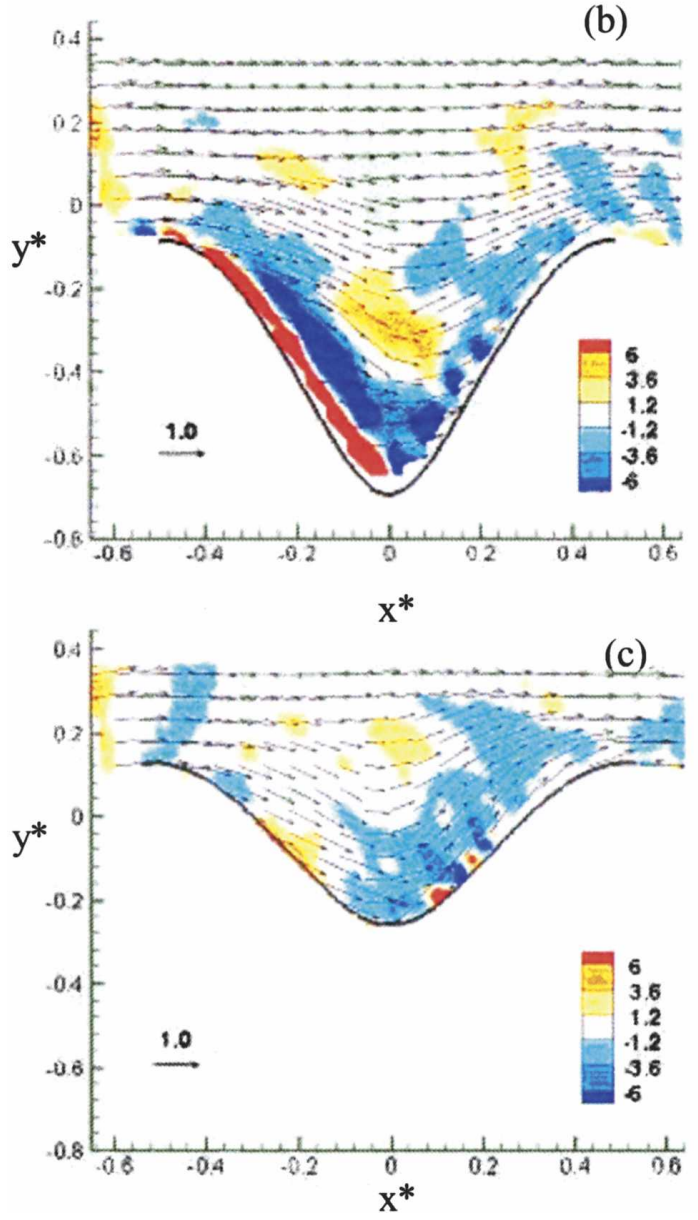

(d)

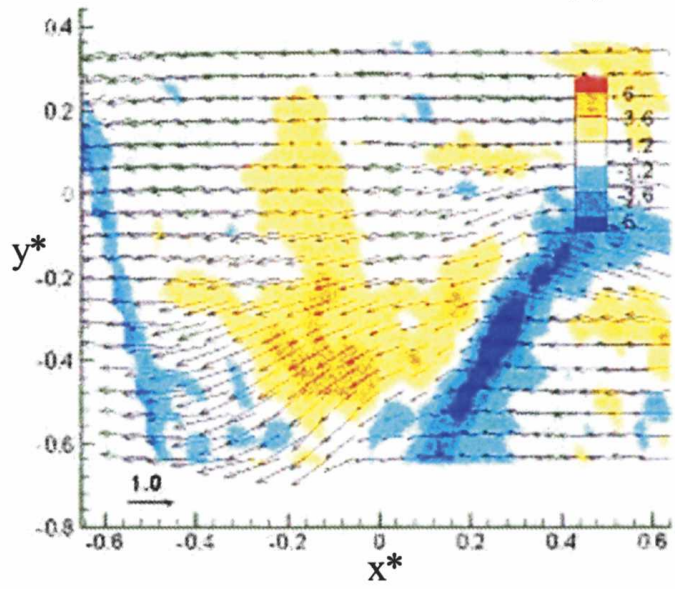

(e)

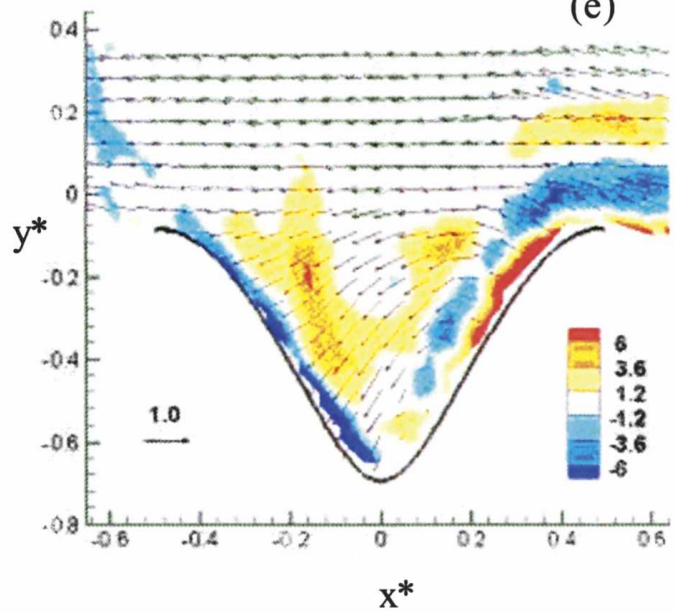

(f)

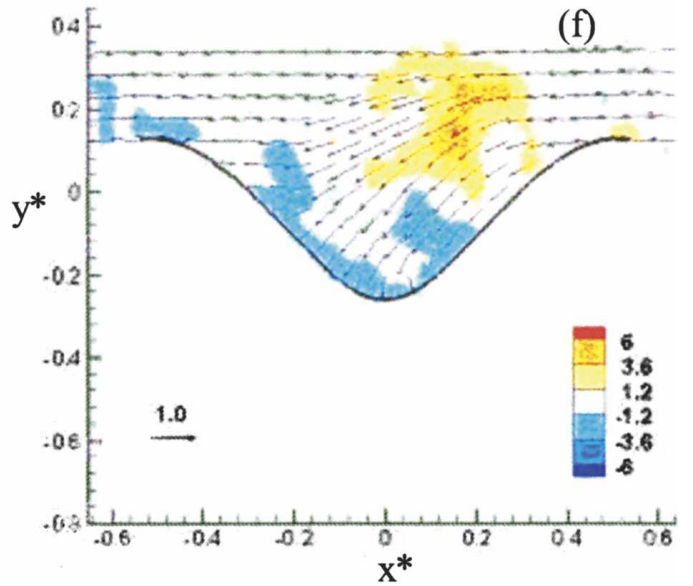

FIG. 4. Phase-averaged velocity and divergence fields for FAST-R experiment for (a), (b), (c) phase A (maximum velocity from left to right) and for (d), (e), (f) phase $\mathrm{C}$ (maximum velocity from right to left). The elevations from top to bottom are (a), (d) $z^{*}=-0.1$; (b), (e) $z^{*}=-0.2$; and (c), (f) $z^{*}=-0.4$.

the development of numerical models of such processes and should be addressed in future studies.

The Reynolds number based on the laminar boundary layer thickness $\operatorname{Re}_{\delta}$ for the FAST experiments is
141. This is well above Lilly's (1966) critical value for stability of a flat Ekman boundary layer and of the same order as that found by Caldwell and Van Atta (1970) for fully developed turbulence. The Reynolds 

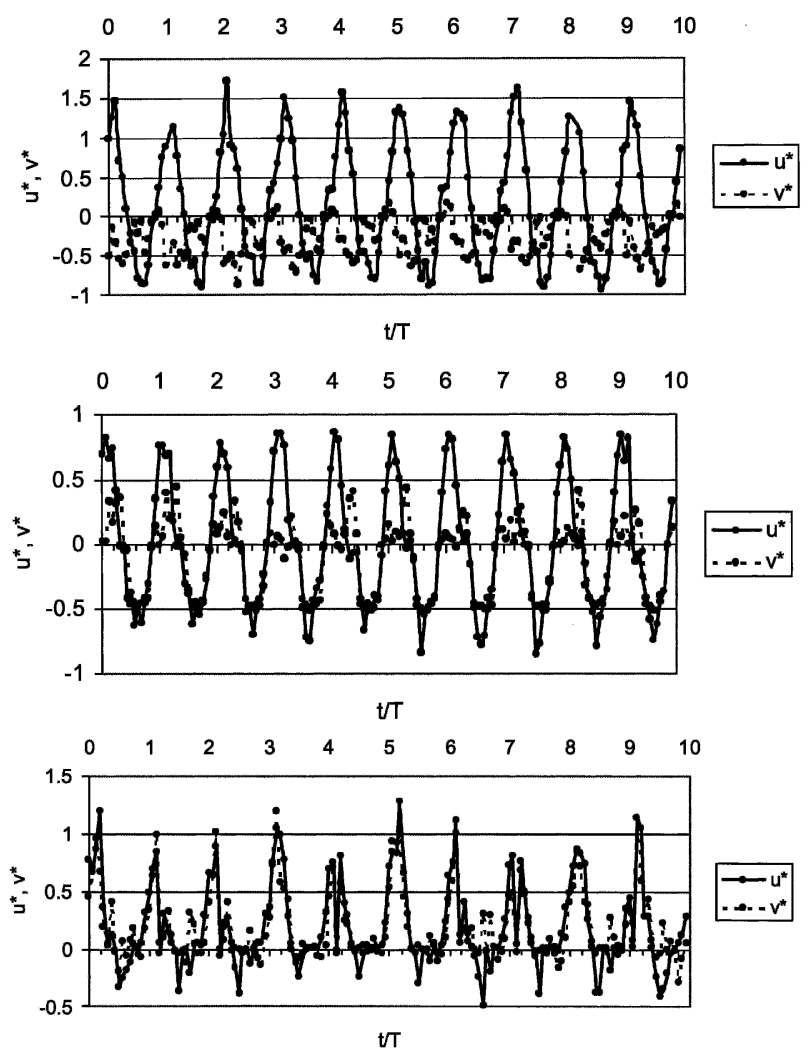

FIG. 5. Normalized time series measurements of the $\left(u^{*}, v^{*}\right)$ velocity components at the shelf break for positions (top) 1 , (middle) 2, and (bottom) 3 (see lower-right insert in Fig. 1) for FAST-R experiment.

number $\mathrm{Re}_{d}$ for the flow past the roughness elements, $\mathrm{Re}_{d}=1500$, is also well above the critical value for turbulence for the flow past cubical elements mounted on a flat surface. The fact that $\mathrm{Re}_{\delta}$ and $\mathrm{Re}_{d}$ are both above their critical values strongly suggests the presence of boundary turbulence during the higher velocity phases of the oscillatory cycle for the FAST-R experiment. Furthermore, the large $\mathrm{Re}_{\delta}$ for the FAST-S experiment and the close correspondence between the interior flows for this and the FAST-R experiment supports the notion that boundary turbulence is also present for the FAST-S run.

While the PIV system had a spatial resolution of only $6.5 \mathrm{~cm}$, the interior motion field nevertheless exhibited large-scale turbulent fluctuations, supporting the premise that the bottom boundary layers were turbulent as well. Figure 6a shows instantaneous velocity and vorticity fields obtained for the FAST-R experiment for phases $\mathrm{A}$ and $\mathrm{C}$ of the oscillatory motion, that is, maximum flow from left to right and maximum flow from right to left, respectively. These figures show a wide range of scales of random eddy structures in the vorticity field from those that can just be resolved by the
PIV to those having the scale of the canyon itself. The instantaneous flow fields indicate the transitory formation of narrow jets in these regions but the spatial resolution was not sufficient to estimate the additional dissipation of these wake effects, in comparison with Ekman friction. Figure $6 \mathrm{~b}$ shows plots of the distribution of turbulence intensity [i.e., $\left(u^{\prime 2}+v^{\prime 2}\right) / u_{0}^{2}$ ] obtained from the same runs as those of Fig. 6a. One notes the strong turbulence along the left bank of the canyon for the left-to-right flow and on the right bank for the rightto-left flow.

\section{c. Time-mean flow}

The time-mean Eulerian velocity field is obtained by taking the mean of the phase-averaged velocity field at each point in the flow field. This phase average was determined from measurements of the second 10 oscillations of the flow at the three levels considered in the experiments. The time-mean velocity and vorticity fields for the FAST-S and the FAST-R experiments at the observation levels at middepth of the shelf water, at the shelf break, and at a depth below the rim are given as examples in Figs. 7a-c and 7d-f, respectively. These figures show that both FAST-S and FAST-R experiments have qualitatively similar velocity and vorticity fields at all levels. The flow near the head and on the right side of the canyon facing the deep water is dominated by cyclonic vorticity, and this extends throughout the fluid column but becomes much weaker above the shelf break. At the shelfbreak level and below, the time-mean flow is seen to follow the canyon depth contours, entering the canyon on the left and leaving on the right. There seem to be no obvious large quantitative differences between the two experiments. A closer look, however, does show some differences as described below.

The observed horizontal speed $\bar{u}_{\text {obs }}\left(z^{*}\right) / u_{0}$ was then determined using relation (2.9) at each observation level for all of the experiments. Figures $8 \mathrm{a}, \mathrm{b}$ are plots of $\bar{u}_{\text {obs }}\left(z^{*}\right) / u_{0}$ as a function of the normalized vertical coordinate $z^{*}$ for the FAST-R, -S and the SLOW-R, -S and ASU-01 experiments, respectively. The only difference between the FAST-S, -R runs of Fig. 8a and the SLOW-R, -S experiments of Fig. $8 \mathrm{~b}$ is that the $\mathrm{R}$ cases include surface roughness elements. Thus, the larger the surface roughness (or the stronger the boundary turbulence for the FAST experiments) is, other parameters being fixed, the weaker the residual flow is. Increased surface roughness thus tends to decrease the value of $K_{t}$ in (2.7).

Based on the fact that all of the dynamical parameters except the Ekman number are the same for the SLOW-S and the ASU-01 experiments (see Table 2), 
(a)
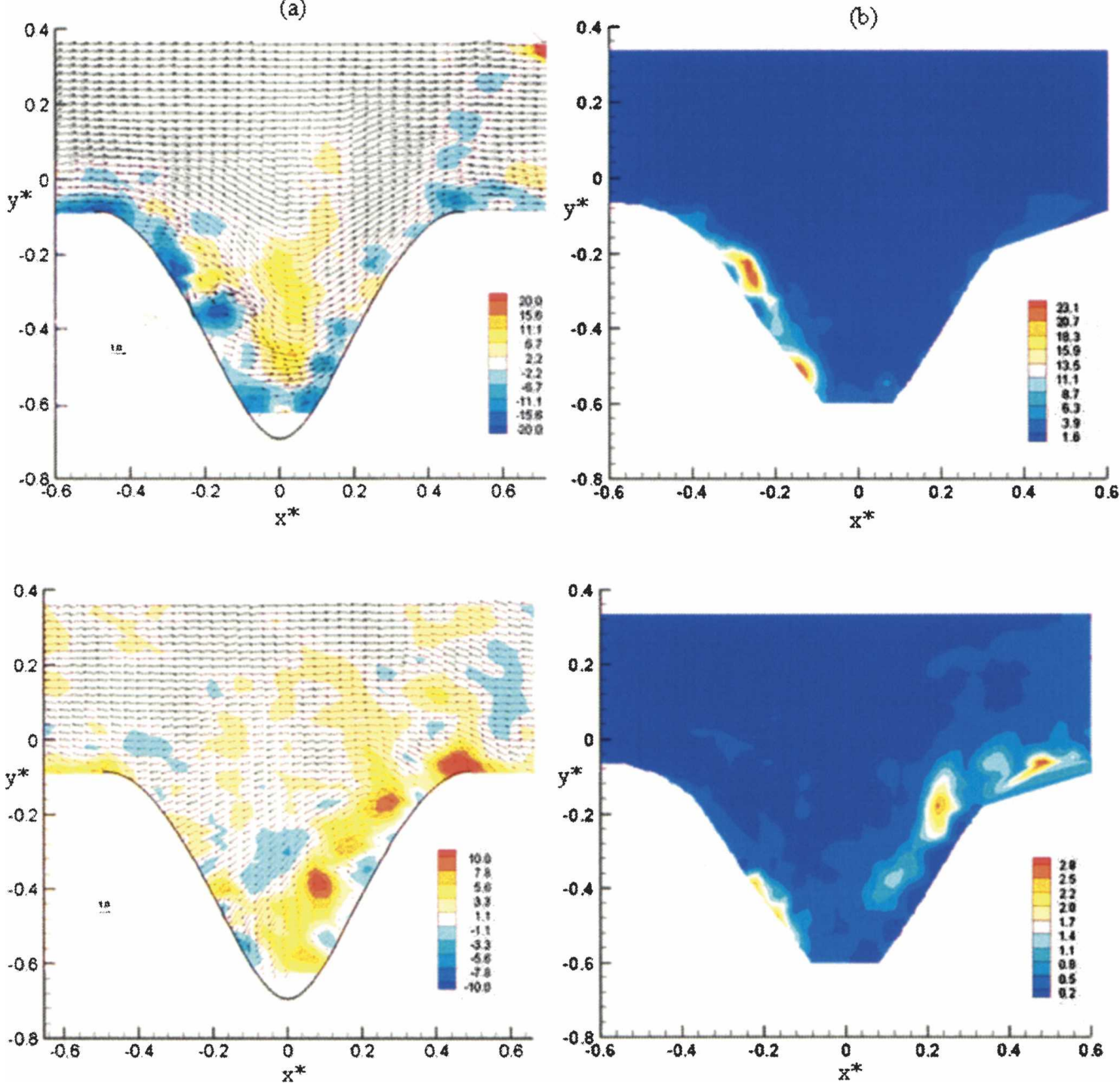

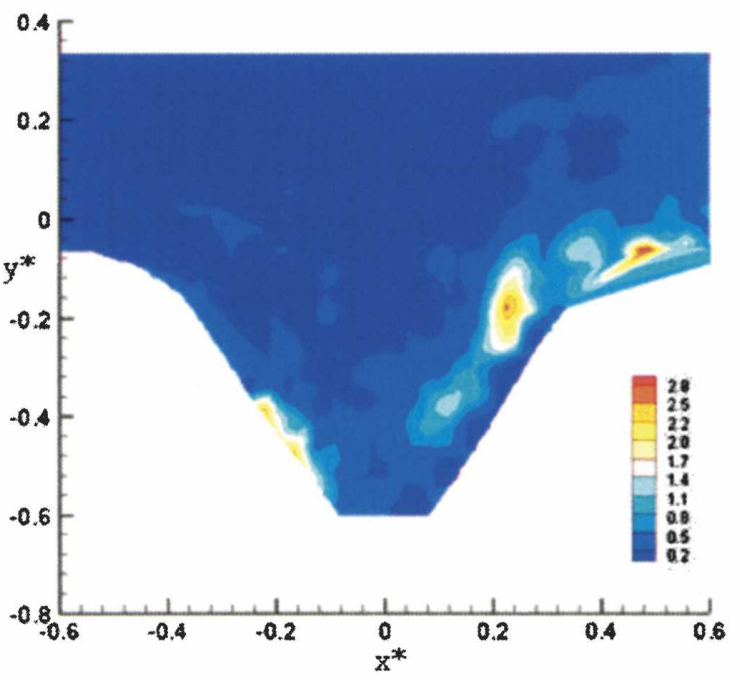

FIG. 6. (a) Instantaneous velocity and vorticity fields at the shelfbreak level for phases A and C. (b) Distribution of the normalized turbulence intensity at the shelfbreak level obtained by phase averaging the PIV data for oscillation cycles 2-20 for phases A and C.

with Ek for the former being roughly one order of magnitude smaller, one expects from the scaling argument that $\bar{u}_{\text {obs }}\left(z^{*}\right) / u_{0}$ for the latter would be less than the former, at all levels. The data support this hypothesis at the upper observation levels and, considering the error bars, with each being approximately the same at the lowest level.

We next investigate the behavior of the characteristic horizontal velocity as evaluated at the shelfbreak level $\bar{u}_{\text {obs }}(-0.2) / u_{0}$ as a function of the scaling parameter. Figure 9 is such a plot, where $\bar{u}_{\text {obs }}(-0.2) / u_{0}$ has been multiplied by the factor $\sqrt{2} / 2$ so as to allow the present experiments to be plotted with the same abscissa as those of BHP. One notes from Fig. 9 that the charac- teristic velocity for the FAST experiments is the same as that obtained for a value of the parameter lambda roughly 6 times that of the fit from the laminar experiments. Therefore, we have definitely reached a turbulent regime, which scales very differently than the previous laminar experiments. Assuming the scaling law (2.7), coefficients $K_{t} \approx(0.49,0.46)$ are obtained for the smooth and rough topographies, respectively. Note, however, that more experiments would be needed to test the scaling law (2.7) itself.

We now address the issue of the "local" mean transport of fluid as driven by the oscillatory motion past the canyon. If one focuses on a control volume that encompasses an isolated topographic feature such as a canyon, 
(a)

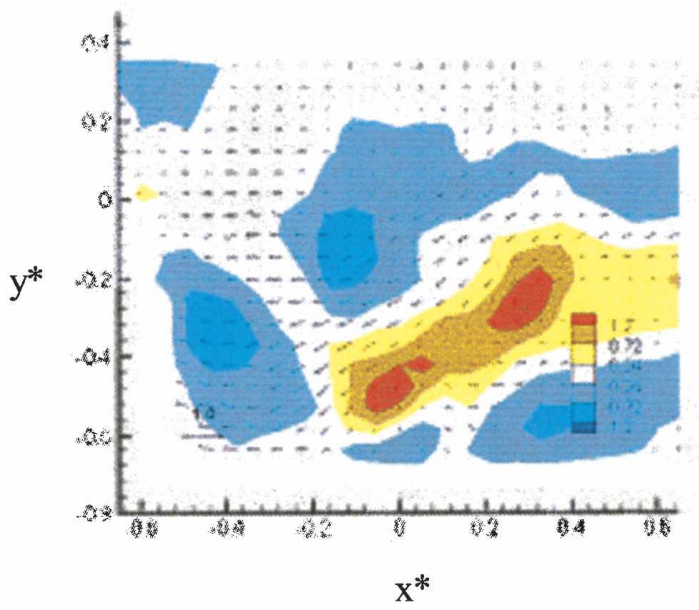

(b)

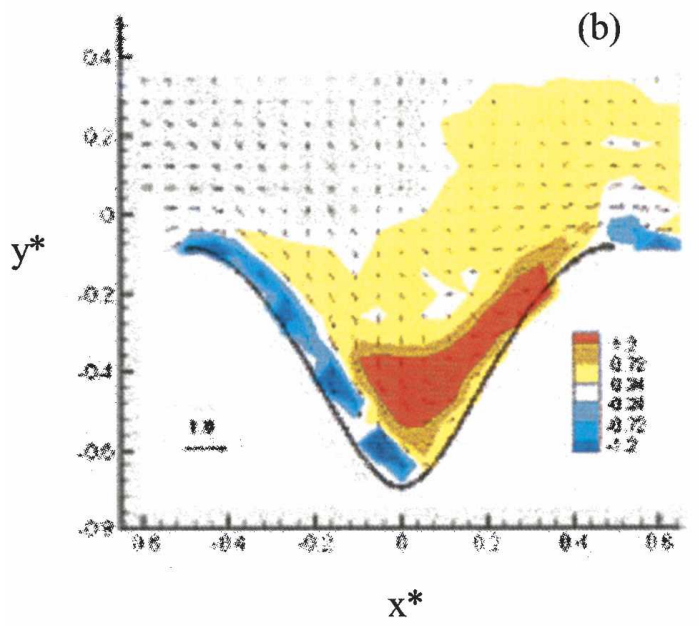

(c)

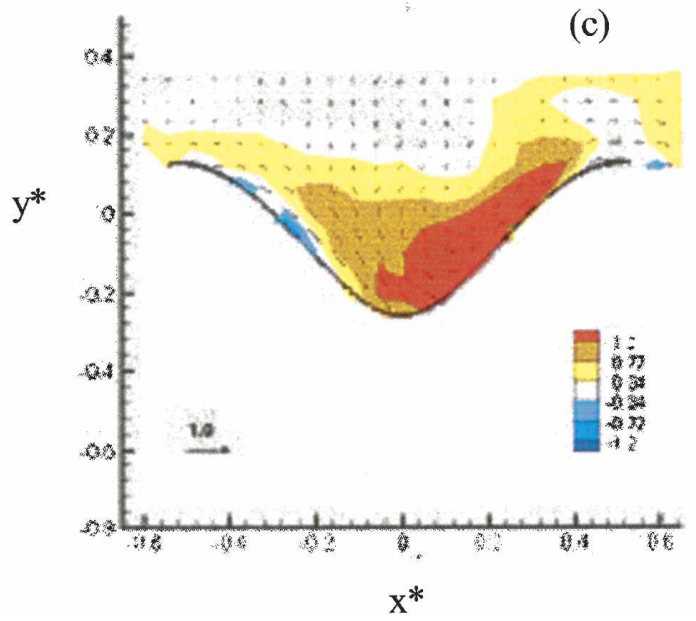

(d)

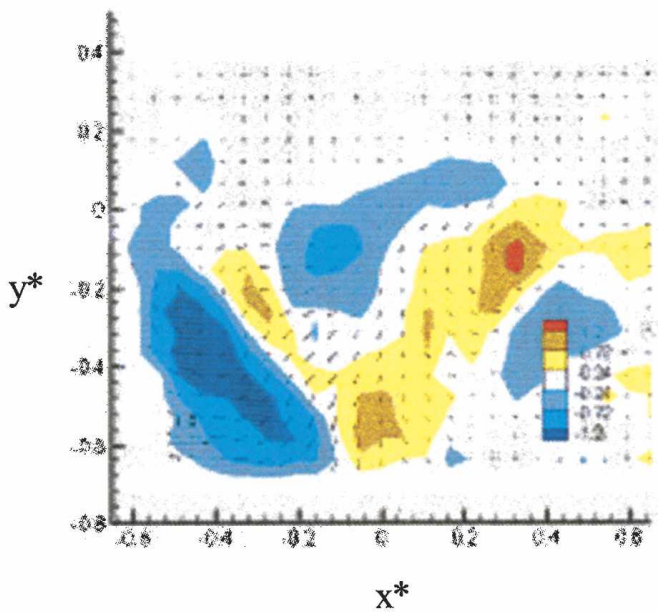

(e)

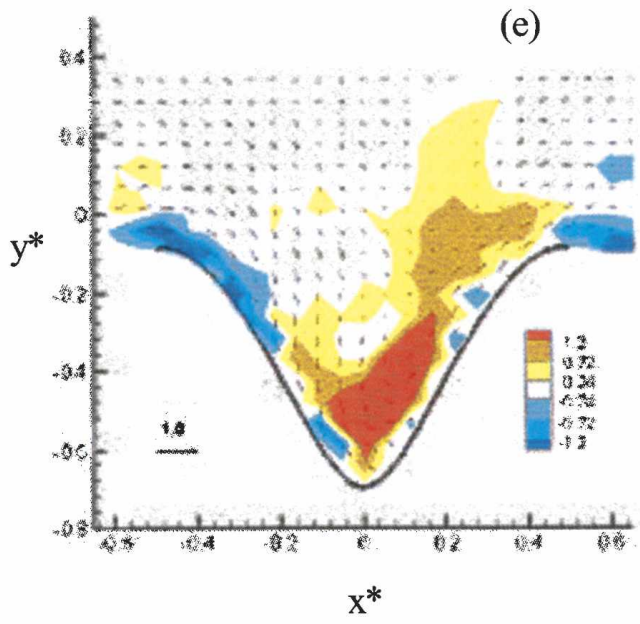

(f)

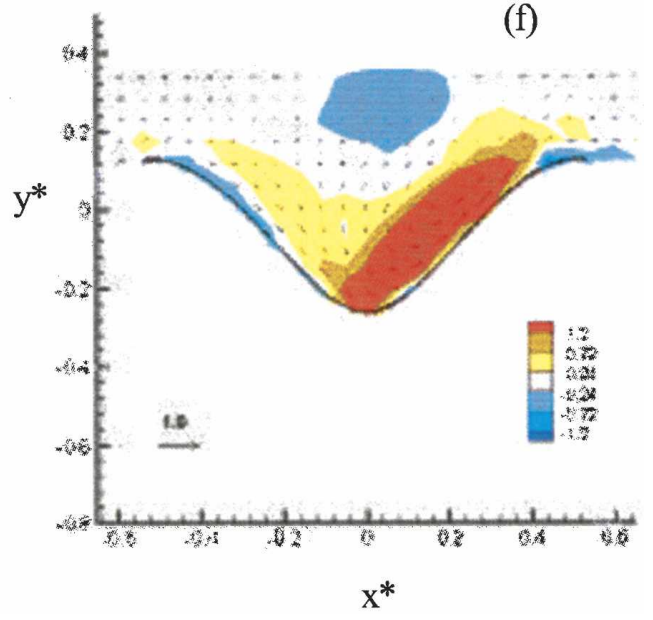

FIG. 7. Time-mean velocity and vorticity fields for the FAST-S and FAST-R experiments at the observation levels at

(a), (d) middepth of the shelf water, $z^{*}=-0.1$; (b), (e) shelf break, $z^{*}=-0.2$; and (c), (f) canyon $\left(z^{*}=-0.4\right)$. 


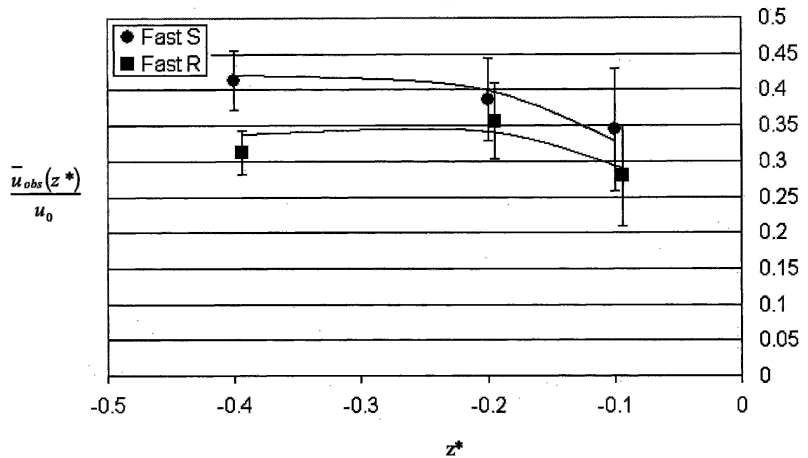

(b)

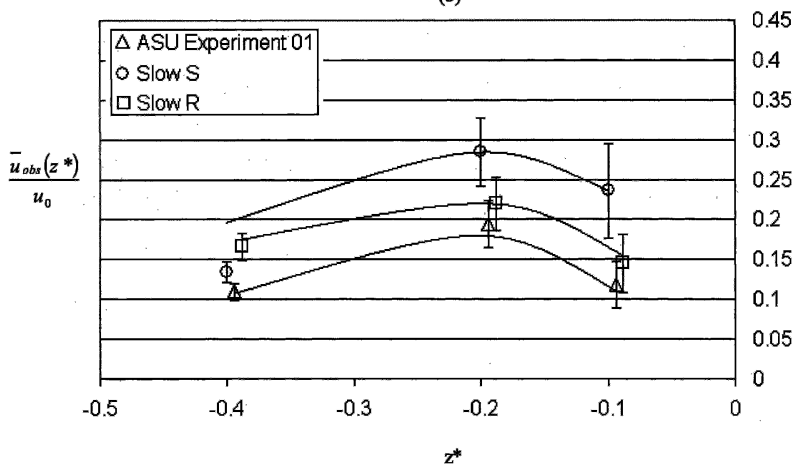

FIG. 8. Characteristic normalized horizontal time-mean velocity $\bar{u}_{\text {obs }}\left(z^{*}\right) / u_{0}$ for (a) FAST-S, -R experiments and (b) SLOW-S, -R and ASU-01 experiments. See Table 2 for parameter values.

conservation of mass requires that the net transport of mass through the surface of the control volume must be zero (e.g., see Fig. 3). The nature of this circulation, however, can play an important role in the local environment.

In the experiments, the normalized volume flux into the control volume through the canyon mouth $Q_{1}^{*}$ was measured at the elevations $z^{*}=-0.1,-0.2,-0.4$, and $Q_{2}^{*}$ and $Q_{3}^{*}$ were measured at $z^{*}=-0.1$. The net upwelling $Q_{z^{*}}^{*}\left(z^{*}\right)$ through each cross-sectional area of the control volume can then be found from (2.13) by integrating from the tank floor to the level in question; the results for the FAST-S, -R and the SLOW-S, -R and ASU-01 experiments are given in Figs. 10a,b, respectively.

In these calculations it has been assumed that on physical grounds the volume flux $Q_{z^{*}}^{*}\left(z^{*}\right)$ must vanish at $z^{*}=-1$ and $z^{*}=0$. Data were obtained only at the points $z^{*}=-0.1,-0.2$, and -0.4 . While only the points for which data were obtained are indicated on the plots, the curve fits themselves were determined by employing extrapolated data between $z^{*}=-0.2$ and $=-0.4$ to obtain $Q_{z^{*}}^{*}(-0.3)$ and between $z^{*}=-0.4$ and the bottom to obtain $Q_{z^{*}}^{*}\left(z^{*}\right)$ near the bottom.

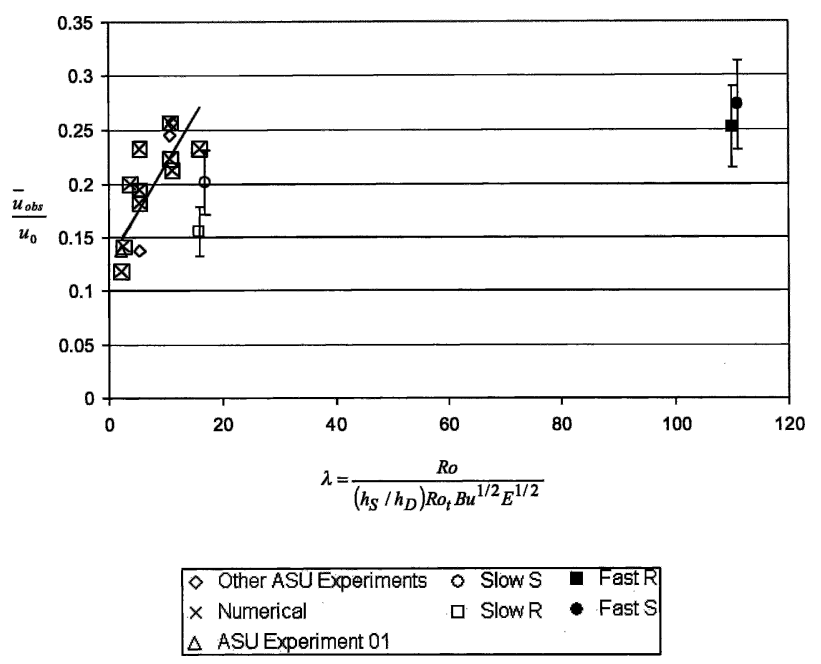

FIG. 9. Characteristic normalized horizontal time-mean speed (multiplied by the factor $1 / \sqrt{2}$ to relate to the BHP definition), evaluated at the shelfbreak level against the scaling parameter $\lambda$. The data from BHP and the best-fit straight line through those data are indicated.

One concludes from Figs. 10a and 10b, and the earlier numerical and physical experiments of PHB and BHP, that upwelling below the shelf break is a ubiquitous feature of these oscillatory forced flows for laminar, transitional, and turbulent flows. Starting at the model floor, the net upwelling for each experiment increases monotonically until reaching a maximum in the vicinity of the shelf break. The data further show that the net flow through the canyon mouth at the midshelfdepth level $Q_{1 *}^{*}\left(z^{*}=-0.1\right)$ is into the canyon, and thus the upwelled flux and the inflow from the deep water above the shelf break is balanced by a flow out of the canyon region across the shelf break along both of its flanks; that is, $\left(Q_{2}^{*}, Q_{3}^{*}\right)$ are both negative. The upwelled water then passes over the shelf break and away from the canyon along its flanks. By inference, this transport away from canyon is retuned from the shelf to the deep ocean.

Figure 10a shows that the higher boundary turbulence (higher boundary drag) experiment exemplified by the FAST-R experiment, other parameters being fixed, develops less upwelling in the lower region (corresponding to less transport into the canyon through its mouth) than does its lower turbulence counterpart (FAST-S). Similarly, Fig. 10b shows that the increased drag in the SLOW-R experiment also leads to a smaller upwelling flux below the shelf break than does its lower-drag counterpart SLOW-S, with other parameters being fixed. There is, however, no significant influence of roughness near the shelfbreak level. This is in agreement with the estimates (2.8), in which friction 
(a)

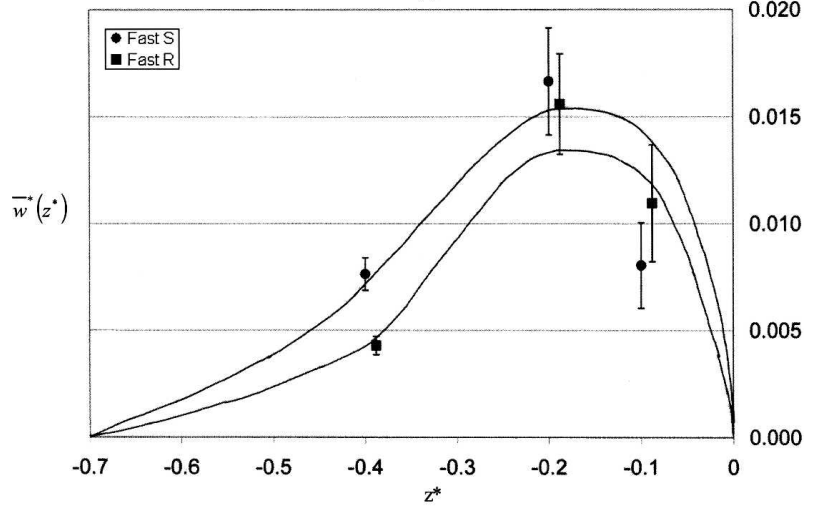

(b)

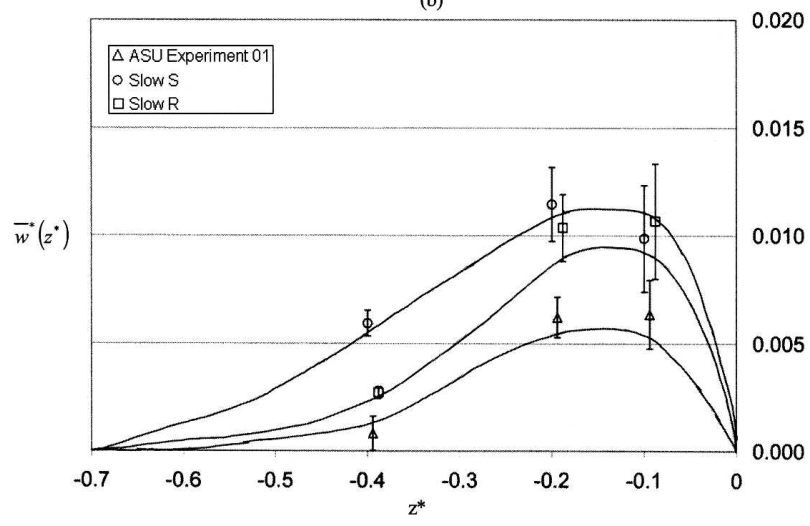

FIG. 10. Characteristic, normalized time-mean upwelling speed plotted against the normalized vertical coordinate $z^{*}$ for (a) FAST-S, -R experiments and (b) SLOW-S, -R and ASU-01 experiments. See Table 2 for parameter values.

does not appear. We also expect from (2.8) a good coincidence between SLOW and ASU-01 results on upwelling, since the parameter $u_{0} /(N L)$ is the same in both experiments. There is, however, less upwelling in ASU01 results, as seen in Fig. 10b. In comparing SLOW and FAST experiments (Figs. 10a,b), we note that the relative upwelling increases with the forcing $u_{0}$, although not as strongly as predicted by (2.8).

The final issue to be addressed is that of the differences (errors) involved in the measurements taken in the laboratory experiment leading to Figs. 8, 9, and 10. The wind shear stress on the fluid surface due to the rotation of the test cell in the quiescent laboratory air was identified as the principal source of these differences. To consider this issue a series of experiments was conducted in which the fluid in the test cell (with topography) was driven by wind stress only. The velocity field over the canyon was monitored as in the regular modulation experiments. These data were then phase and time averaged to determine $(u, v)$ and hence $\bar{u} / u_{0}$ and $Q_{1}^{*}, Q_{2}^{*}$, and $Q_{3}^{*}$ for each level considered in the regular experiments; the data were time averaged for 10 equivalent oscillation cycles.

Denoting the wind-driven-only experiments by the symbol $W$ and the regular or modulated experiments by $M$, the ratios of the characteristic horizontal speeds $\bar{u}_{W} /$ $\bar{u}_{M}$ and volume transports $Q_{W i}^{*} / Q_{M i}^{*}$ were then determined at each measurement level and for each of the experiments. These ratios were similar for both the horizontal speed and the horizontal transport and varied with depth, being substantially larger at the upper levels and for the SLOW runs. The error bars in Figs. 8, 9 , and 10 are obtained from these data and are estimated for the respective levels $\left(z^{*}=-0.1,-0.2,-0.4\right)$ as SLOW $\pm(0.5,0.3,0.1)$ and FAST $\pm(0,3,0.15,0.10)$, each fraction multiplied by the level of the signal.

\section{Summary, discussion, and concluding remarks}

This study has demonstrated that the qualitative features of canyon flows forced by oscillatory along-shelf currents, whose boundary layers are turbulent or transitional during at least part of each flow cycle, are qualitatively similar to their laminar counterparts as found earlier by PHB and BHP. For example, phase-averaged velocity fields, owing to rotation effects, are shown to be asymmetric when the phase of the current has the coastline on its left and when it is on its right. Furthermore, the nature of the asymmetries for the turbulent and transitional flows mirrors that of the laminar ones.

These similarities are also apparent for the respective time-mean flows and include the dominance of cyclonic vorticity in the canyon interior with the location of the maximum being near the head of the canyon and along its right side facing deep water, an along-shelf current with the coastline on the right following the depth contours of the canyon, and a mean upwelling in the canyon that increases with elevation becoming a maximum near the shelf break and with the upwelled fluid being advected away from the canyon onto the shelf on both flanks of the canyon; a schematic diagram of the timemean circulation is given in Fig. 11.

The SLOW and FAST experiments both reveal clear quantitative differences with respect to the laminar scaling law for the residual flow, supporting the claim that these experiments are transitional and turbulent, respectively. Although we were not able to measure near-wall turbulence directly in our experimental system, we do observe turbulent fluctuations at larger scale, associated with boundary layer detachment. We have extended the scaling model (2.5) of (BHP) to the case of a more general wall-friction law for the timeaveraged characteristic speed. In particular, assuming that bottom friction is controlled by turbulent fluctua- 


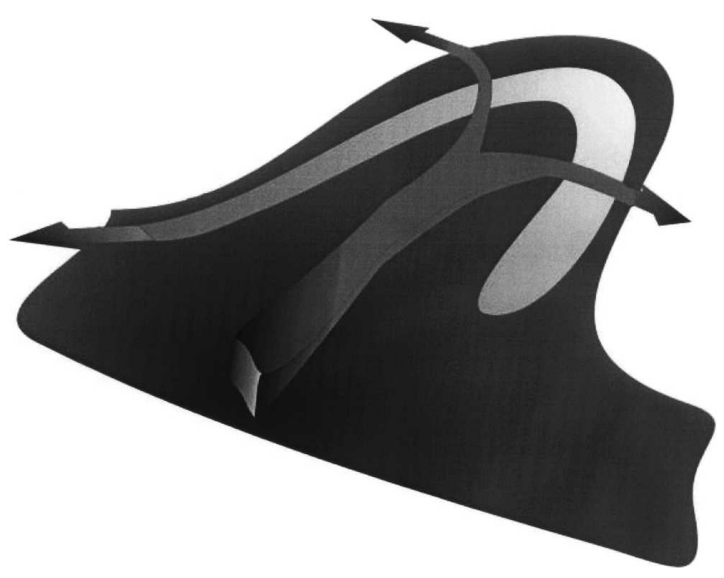

FIG. 11. Interpretive sketch of typical time-mean flow.

tions that scale as the forcing velocity $u_{0}$, we have derived scaling laws for 1) the time-mean horizontal velocity $[(2.7)]$ and 2) the upwelling velocity. The coefficients $K_{t} \approx(0.49,0.46)$ for the FAST-S, -R experiments, respectively, are found from (2.7). Only one set of parameters (FAST experiments), however, is available in the turbulent regime. We thus cannot test the scaling laws themselves.

Hickey (1997) found that upwelling within Astoria canyon "is simultaneous and spatially uniform." The laboratory experiments, on the other hand, show significant spatial and temporal variability. Hickey also points out that the estimated vertical velocities within the canyon were on the order of $50 \mathrm{~m} \mathrm{day}^{-1}$. Using the upwelling data obtained in the laboratory and depicted in Fig. 10a, at the shelfbreak level, say, and using the appropriate coordinate transformations, the spatially averaged upwelling for Astoria-like parameters is $4 \mathrm{~m}$ day $^{-1}$. It is suggested that the principal reason for the large difference between the laboratory and the ocean upwelling estimates is that the laboratory value is an average for the entire canyon. On the other hand, the intense upwelling is concentrated in small regions of the canyon as evidenced, for example, by the horizontal divergence fields depicted in Fig. 4.

Although the laboratory results have demonstrated a number of flow characteristics that have their counterparts in the ocean, reaching the long-term goal of providing numerical modelers with benchmark data to develop and test their models will require that the laboratory studies focus on a number of issues raised in this study. The first is the need either to eliminate the shear stress applied to the atmosphere-fluid interface or to control and monitor the shear stress during the conduct of the experiments. The second is the need to investigate the details of the bottom boundary layer (e.g., Reynolds stresses) and relate the same to the motion fields developed outside these layers. Because of the complex geometry and periodic forcing employed in the present study, this configuration is not the ideal one to begin studying the effects of surface slope, stratification, and rotation on the bottom boundary layer. Nevertheless, it is fair to say that PIV and other modern techniques of measuring fluid motions in the laboratory have revolutionized fluid mechanics research and have placed laboratory experimentation in a substantially better position to support the development of improved numerical models of the ocean environment.

Acknowledgments. The authors thank the Office of Naval Research (ONR) for support of this research under Grant N00014-01-1-0296. We also appreciate the support of the National Science Foundation (NSF) under Grant OCE-0137197. Special thanks are also given to the two anonymous referees who made numerous suggestions in an earlier version that ultimately led to a far better communication. The first author is grateful to the University of Grenoble, France, for supporting his sabbatical leave during the period January-April 2002, during which time some of the work on this research was being conducted. The experimental work has been funded by the European Commission under Grant HPRI-1999-CT-00006 for "Access to Major Research Infrastructures." Special thanks are also given to Henri Didelle and Rene Carcel for their tireless efforts in conducting the experiments at the Coriolis Laboratory in Grenoble.

\section{REFERENCES}

Boyer, D. L., X. Zhang, and N. Pérenne, 2000: Laboratory observations of rotating stratified flow in the vicinity of a submarine canyon. Dyn. Atmos. Oceans, 31, 47-72.

— D. B. Haidvogel, and N. Pérenne, 2004: Laboratory-numerical model comparisons of canyon flows: A parameter study. J. Phys. Oceanogr., 34, 1588-1609.

Caldwell, D. R., and C. W. Van Atta, 1970: Characteristics of Ekman boundary layer instabilities. J. Fluid Mech., 44, 79-95.

Ferrero, E., and Coauthors, 2005: Physical simulations of neutral boundary layer in rotating tank. Il Nuovo Cimento, 28C, $1-17$.

Hickey, B., 1997: The response of a steep-sided, narrow canyon to time-variable wind forcing. J. Phys. Oceanogr., 27, 697-726.

Lilly, D. K., 1966: On instability of Ekman boundary flow. J. Atmos. Sci., 23, 481-494.

Pérenne, N., D. B. Haidvogel, and D. L. Boyer, 2001: Laboratorynumerical model comparisons of flow over a coastal canyon. J. Atmos. Oceanic Technol., 18, 235-255. 